\title{
RESPOSTA DO TOMATEIRO À FERTIRRIGAÇÃO POTÁSSICA E COBERTURA PLÁSTICA DO SOLO ${ }^{1}$
}

\author{
REGYNALDO ARRUDA SAMPAIO², PAULO CEZAR REZENDE FONTES e CARLOS SIGUEYUKI SEDIYAMA
}

\begin{abstract}
RESUMO - O objetivo do presente estudo foi determinar os efeitos da fertirrigação potássica e da cobertura do solo em película de plástico preto, na cultura do tomate. O experimento foi realizado na Universidade Federal de Viçosa, em solo Podzólico Vermelho-Amarelo câmbico. Os tratamentos, em cinco repetições, no delineamento em blocos casualizados, corresponderam a: (A) aplicação manual de $40 \%$ da dose recomendada de $\mathrm{K}$ no sulco de transplante e $60 \%$ aplicados manualmente, em cobertura; (B) aplicação manual de $40 \%$ da dose de $\mathrm{K}$ no sulco de transplante, e $60 \%$ aplicados por fertirrigação; (C) procedimento idêntico ao anterior, porém com o solo coberto por plástico preto; (D) aplicação de $100 \%$ da dose de K por fertirrigação e (E) procedimento idêntico ao anterior, porém com o solo coberto por plástico preto. Os tratamentos B, C, D e E foram irrigados por gotejamento. Maiores produções de tomates foram obtidas com a aplicação do K por fertirrigação do que com a aplicação manual. Estas produções, entretanto, não foram influenciadas pela aplicação total ou parcial de $\mathrm{K}$ por fertirrigação, nem pela presença de cobertura plástica do solo. Os teores de $\mathrm{N}_{-} \mathrm{NO}_{3}{ }^{-}$, $\mathrm{N}$-orgânico, $\mathrm{K}$, Ca e Mg no pecíolo do tomateiro não foram influenciados pelos tratamentos.
\end{abstract}

Termos para indexação: tomate, adubação potássica, Lycopersicon esculentum.

\section{TOMATO RESPONSE TO POTASSIUM FERTIRRIGATION AND BLACK POLYETHYLENE COVER}

\begin{abstract}
The aim of this work was to determine the effects of fertirrigation with potassium and black polyethylene cover on tomato. A field experiment was carried out on a cambic yellowish podzolic at the Universidade Federal de Viçosa, in Viçosa, MG, Brazil. The treatments, with five replicates, following a randomized block design, were: (A) manual application of $40 \%$ of recommended $\mathrm{K}$ rate at the seedling transplantation row and manual sidedress application of $60 \%$; (B) manual application of $40 \%$ of recommended $\mathrm{K}$ rate at the seedling transplantation row and $60 \%$ by fertirrigation; (C) similar procedure to B but with black polyethylene cover; (D) application of $100 \%$ of $\mathrm{K}$ rate by fertirrigation and (E) similar procedure to D but with black polyethylene cover. Treatments B, C, D and E were drip irrigated. Tomato yields were higher with $\mathrm{K}$ application by fertirrigation than using the manual fertilization method, but the tomato yields were not influenced by $\mathrm{K}$ partial or total fertirrigation neither by black polyethylene cover. The $\mathrm{NO}_{3}^{-}-\mathrm{N}$, organic- $\mathrm{N}, \mathrm{K}, \mathrm{Ca}$ and $\mathrm{Mg}$ concentrations on leave petioles were not influenced by treatments.
\end{abstract}

Index terms: tomato, potassic fertilization, Lycopersicon esculentum.

\section{INTRODUÇÃO}

A aplicação de fertilizantes via água de irrigação constitui prática importante na cultura do tomatei-

\footnotetext{
${ }^{1}$ Aceito para publicação em 13 fevereiro de 1998. Extraído da Tese de Doutorado do primeiro autor, apresentada à UFV.

2 Eng. Agr., D.Sc., Prof. Adjunto, CCA/UFRR. CEP 69306-210 Boa Vista, RR. Bolsista do CNPq. E-mail: rsampaio@mandic.com.br

${ }^{3}$ Eng. Agr., Ph.D., Prof. Titular, DFT/UFV, CEP 36571-000 Viçosa, MG. Bolsista do CNPq.
}

ro. Esta técnica proporciona aumento na disponibilidade de nutrientes, reduz os custos com mão-de-obra, melhora a distribuição do adubo no campo e facilita seu parcelamento (Locascio \& Myers, 1974; Steduto, 1984).

A aplicação da dose total de K, no momento do transplante do tomateiro, pode aumentar a concentração salina em torno das raízes, aumentar as perdas deste nutriente por lixiviação e reduzir a eficiência de sua utilização pelas plantas. Também, em razão do pequeno desenvolvimento radicular, a aplicação da dose inicial de K via irrigação localizada 
no momento do transplante pode movimentar os íons para a extremidade do bulbo molhado, dificultando a absorção dos nutrientes pelas plantas (Fischer, 1992; Hartz, 1994). Nessas condições, Locascio et al. (1982) e Dangler \& Locascio (1990) recomendam que por ocasião do transplante, entre 30 e $40 \%$ das doses recomendadas de $\mathrm{K}$ sejam incorporadas ao solo e o restante seja aplicado por fertirrigação durante o ciclo da cultura.

A utilização da fertirrigação é compatível com a cobertura do solo por plástico, prática comum na produção de tomate para mesa nos EUA (Baki-Abdul \& Spence, 1992). A cobertura de plástico protege a planta contra pragas do solo, controla a incidência de plantas daninhas, conserva a umidade próxima à superfície do solo, aumenta a concentração de raízes na parte mais aquecida e mais fértil do perfil do solo, aumenta a atividade microbiana e a taxa de mineralização do N orgânico e, principalmente, evita a lixiviação de nitrato e potássio, importantes para a nutrição do tomateiro (Clark \& Maynard, 1992; Tsekleev et al., 1993). Estudos empregando a combinação de cobertura do solo com plástico e fertirrigação potássica do tomateiro, embora inexistentes na literatura nacional, são difundidos na literatura estrangeira (Fischer, 1992).

O objetivo deste trabalho foi determinar os efeitos da fertirrigação parcial ou total com potássio e da cobertura do solo com plástico sobre o tomateiro.

\section{MATERIAL E MÉTODOS}

O experimento foi realizado na Horta do Fundão da Universidade Federal de Viçosa, em solo Podzólico Vermelho-Amarelo câmbico com as características químicas e físicas apresentadas na Tabela 1 .

Sementes de tomate (Lycopersicon esculentum, Mill.), cv. Santa Clara, foram semeadas em sementeira, em 8 de setembro de 1995. Nessa mesma data, no solo onde seriam transplantadas as mudas, foram incorporados até $50 \mathrm{~cm}$ de profundidade, $40 \%$ da dose de calcário dolomítico necessária para elevar a porcentagem de saturação de bases para $70 \%$. Os $60 \%$ restantes foram incorporados até $15 \mathrm{~cm}$ de profundidade, três dias antes do transplante, em 27 de setembro de 1994

Em todos os tratamentos, um percentual de $40 \%$ do fertilizante potássico foi colocado no momento do transplante, e os $60 \%$ restantes foram aplicados em cobertura, em três vezes, a cada 15 dias, a partir da adubação realizada no momento do transplante. No tratamento A, considerado testemunha, o fertilizante foi aplicado manualmente no sulco de transplante e, também manualmente, em cobertura ao lado das plantas, com irrigação por aspersão no solo, com mangueira. Nos tratamentos B e C (fertirrigação parcial), o adubo potássico foi colocado manualmente no sulco de transplante, e as aplicações em cobertura foram feitas via água de irrigação. Nos tratamentos D e E (fertirrigação total), o adubo potássico foi aplicado sempre via água de irrigação, tanto no momento do transplante quanto nas aplicações em cobertura. Os tratamentos $\mathrm{C}$ e $\mathrm{E}$ receberam cobertura plástica do solo. Cada um dos cinco tratamentos estudados ocupou uma parcela de $14 \mathrm{~m}^{2}$, com espaçamento de $0,5 \mathrm{~m}$ entre plantas e $1,0 \mathrm{~m}$ entre fileiras e com 10 plantas úteis. Os tratamentos foram distribuídos no delineamento em blocos casualizados com cinco repetições.

A dose de $\mathrm{K}$, aplicada na forma de $\mathrm{KCl}$, foi equivalente a $186 \mathrm{~kg} \cdot \mathrm{ha}^{-1} \mathrm{e}$ correspondeu à dose de máxima eficiência física da produção comercial de tomate obtida por Sampaio (1996). O N foi aplicado na forma de uréia, em doses equivalentes a $80 \mathrm{~kg} \cdot \mathrm{ha}^{-1} \mathrm{de} \mathrm{N}$, no sulco de transplante, e $120 \mathrm{~kg} \cdot \mathrm{ha}^{-1}$ de $\mathrm{N}$ parcelado em três vezes, nas mesmas datas em que o adubo potássico foi parcelado. Por ocasião do transplante, foram aplicados, também no sulco, $180 \mathrm{~kg} \cdot \mathrm{ha}^{-1}$ de $\mathrm{P}_{2} \mathrm{O}_{5}$, na forma de superfosfato simples, 10 kg.ha ${ }^{-1}$ de bórax, 10 kg.ha-1 de sulfato de zinco e 200 g.ha1 de molibdato de amônio, conforme recomendação da Comissão de Fertilidade do Solo do Estado de Minas Gerais (1989).

TABELA 1. Características químicas e físicas do soloํ.

\begin{tabular}{|c|c|c|c|c|c|c|c|c|c|c|}
\hline $\begin{array}{c}\mathrm{C} \\
\left(\text { dag. }^{-1} \mathrm{~kg}^{-1}\right)\end{array}$ & $\begin{array}{c}\mathrm{pH}-\mathrm{H}_{2} \mathrm{O} \\
1: 2,5\end{array}$ & $\begin{array}{c}\mathrm{P} \\
----(\mathrm{mg} . \mathrm{dm}\end{array}$ & $\begin{array}{c}\mathrm{K}^{+} \\
\left.\mathrm{n}^{-3}\right) \\
\end{array}$ & $\mathrm{Ca}^{2+}$ & $\begin{array}{c}\mathrm{Mg}^{2+} \\
--(\mathrm{cmo}\end{array}$ & $\begin{array}{c}\mathrm{Al}^{3+} \\
\left(\mathrm{m}^{-3}\right)\end{array}$ & $\mathrm{H}+\mathrm{Al}$ & Argila & $\begin{array}{l}\text { Silte } \\
\text { g.kg }\end{array}$ & Areia \\
\hline 1,87 & 5,6 & 60,3 & 58,5 & 3,2 & 0,6 & 0,1 & 4,2 & 50 & 14 & 36 \\
\hline
\end{tabular}

${ }^{1} \mathrm{P}$ e K: extrator Mehlich-1; $\mathrm{Al}$, Ca e Mg: extrator $\mathrm{KCl} 1 \mathrm{~mol} \mathrm{~L}^{-1}$; $\mathrm{H}+\mathrm{Al}$ : extrator $\mathrm{Ca}(\mathrm{OAc}), 0,5 \mathrm{~mol} \mathrm{~L}^{-1}$ a pH 7,0. 
Nos tratamentos com cobertura plástica, o solo foi coberto quinze dias após o transplante das mudas, com película de polietileno preto de baixa densidade medindo 3,6 m de comprimento por 1,2 $\mathrm{m}$ de largura. As bordas da película entre as fileiras foram superpostas e cobertas com solo, como em outros países que utilizam a cobertura do solo com plástico (Salvetti, 1985).

O tomateiro foi conduzido com duas hastes tutoradas no sistema de cerca cruzada. A poda apical foi realizada quando a planta atingiu $1,8 \mathrm{~m}$ de altura.

A demanda de água pela planta foi estimada com base na evaporação de tanque classe $\mathrm{A}$, considerando o coeficiente do tanque, equivalente a 0,85 , e os coeficientes de cultura variáveis dependendo do estádio de desenvolvimento do tomateiro (Volpe \& Churata-Masca, 1988). No tratamento A, a irrigação foi realizada no sulco com mangueira, utilizando-se hidrômetro e chuveiro adaptados na extremidade (Soares, 1986). Nos demais tratamentos, a irrigação foi realizada por gotejamento, pelo método xique-xique (Gonçalves, 1988), com as linhas laterais distribuídas entre fileiras, e com a saída de água a $10 \mathrm{~cm}$ de distância da planta. O sistema de irrigação foi instalado com tanque de 20,4 litros para aplicação do fertilizante (Bonomo, 1995); durante a aplicação do K na fertirrigação, o volume de água derivado para o tanque correspondeu a cinco vezes o volume deste. O turno de rega adotado foi variável e a cada dois turnos de rega de dois dias intercalava-se um de três dias. A lâmina de água aplicada durante o ciclo da cultura foi $231,5 \mathrm{~mm}$ em complementação aos $734,8 \mathrm{~mm}$ de chuva $(322,9 \mathrm{~mm}$ do transplante até a primeira colheita mais $411,9 \mathrm{~mm}$ da primeira até a sétima colheita).

Durante cinco turnos de rega, em 13/11/95, 27/11/95, 4/12/95, 18/12/95 e 1/1/96, escolhidos aleatoriamente imediatamente antes da irrigação, foram coletadas amostras de solo até $10 \mathrm{~cm}$ de profundidade, para a determinação da umidade pelo método termogravimétrico. Nessas ocasiões, e na mesma profundidade do solo, foram determinadas as temperaturas: mínima, às 8h; e máxima, às $16 h$.

No florescimento do sexto cacho, foram coletadas amostras do pecíolo da folha completamente expandida mas não-senescente, e do pecíolo da folha adjacente ao sexto cacho, para determinação dos teores de N-orgânico utilizando-se o reagente de Nessler (Jackson, 1958), ${\mathrm{N}-\mathrm{NO}_{3}}^{-}$(Cataldo et al., 1975), Ca, Mg e K na matéria seca (Malavolta et al., 1989).

Os frutos foram colhidos semanalmente, em sete colheitas, quando seus ápices apresentavam coloração avermelhada, e os sem defeitos foram classificados em graúdos $(\varnothing \geq 60 \mathrm{~mm})$, grandes $(56 \mathrm{~mm} \leq \varnothing<60 \mathrm{~mm})$, médios (52 mm $\leq \varnothing<56 \mathrm{~mm})$ e outros $(\varnothing<52 \mathrm{~mm})$. A primeira colheita foi realizada em 7 de dezembro de 1995 , e a última, em 17 de janeiro de 1996

Após a última colheita, foram coletadas amostras de solo nas camadas de 0-10, 10-20, 20-30, 30-40 e 40-50 cm de profundidade, entre plantas, na fileira de plantio, para análise da condutividade elétrica, $\mathrm{pH}$ e teores de $\mathrm{K}, \mathrm{Ca}$,

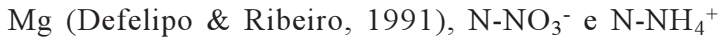
(Tedesco et al., 1985).

A produção comercial de frutos foi obtida por somatório das três primeiras classes. Essas foram consideradas como classes extra AA, extra A e extra, respectivamente. Calculou-se também a produção ponderada de frutos. Para tanto, foram utilizados os fatores de ponderação $1,0,0,6$ e 0,3 , com base nos preços médios das classes supracitadas, verificados nos meses de dezembro de 1995 e de janeiro de 1996 na CEASA-MG, para serem transformadas as classes extra AA, extra A e extra em unicamente extra AA.

Frutos menores do que $52 \mathrm{~mm}$ de diâmetro foram considerados como produção não-comercial. A produção total foi obtida mediante somatório da produção comercial e não-comercial.

Calculou-se o índice de precocidade pela equação $\mathrm{IP}=\sum_{\mathrm{i}=1}^{\mathrm{n}}(\mathrm{Yi} / \mathrm{Di}) / \mathrm{n}$, em que $\mathrm{n}=$ número de colheitas; $\mathrm{i}=1,2, \ldots, \mathrm{n} ; \mathrm{Yi}=$ produção na colheita $\mathrm{i}$; e Di = número de dias do início ao final de cada colheita i (Khanizadeh \& Fanous, 1992).

Os dados obtidos foram submetidos à análise de variância, e os tratamentos, comparados a 5\% de probabilidade pelo teste de Duncan (Pimentel-Gomes, 1987).

\section{RESULTADOS E DISCUSSÃO}

A película de polietileno, dadas as suas características de impermeabilidade, manteve a umidade do solo mais elevada nos intervalos entre as irrigações (Tabela 2). Também, em razão da maior absorção de calor e reirradiação para o solo, as temperaturas máximas e mínimas foram mais elevadas, e os gradientes entre elas foram menores no solo coberto com plástico do que no solo não coberto.

Em geral, as produções total, comercial e ponderada de frutos de tomate foram mais elevadas nos tratamentos irrigados por gotejamento com a fertirrigação, do que no tratamento irrigado por aspersão no solo com mangueira e aplicação manual do fertilizante potássico (Fig. 1). Esse fato poderia estar relacionado à maior eficiência de utilização de 
água, em razão do sistema de irrigação adotado. A irrigação localizada aumenta a concentração de raízes próximas ao gotejador, tornando mais eficiente o uso da água e dos nutrientes.

Na região de Viçosa, as produtividades de tomate em períodos de intensas chuvas e calor raramente ultrapassam 50 t.ha $^{-1}$. No presente experimento, com exceção do tratamento testemunha, os demais apresentaram produções comerciais próximas a 70 t.ha ${ }^{-1}$.

Em relação às produções total, comercial e ponderada de frutos de tomate, nenhuma diferença entre a aplicação parcial e total do K na água de irrigação foi observada (Fig. 1), embora a aplicação do adubo por fertirrigação imediatamente após o transplante possa ser menos eficiente para o tomateiro do que a aplicação manual, em razão do pequeno desenvolvimento radicular neste período e do movimento do $\mathrm{K}$ para as bordas do bulbo molhado (Fischer, 1992; Hartz, 1994). Este fato poderia estar relacionado ao tipo de argila e teor médio de K existente no solo (Tabela 1). O uso da fertirrigação associada à aplicação de lâminas freqüentes de irrigação, comuns em sistemas localizados, podem aumentar (Smajstrla \& Locascio, 1990; Clark et al., 1991), ou não, a produção de tomate (Locascio et al., 1989; Dangler \& Locascio, 1990).

Nas parcelas fertirrigadas, a cobertura do solo com plástico não influenciou as produções total, comercial e ponderada de frutos de tomate (Fig. 1). A

TABELA2. Umidade (U), temperaturas máxima (Tmáx) e mínima (Tmín) e diferença entre Tmáx e Tmín $(\Delta T)$ do solo nos tratamentos: testemunha $(\mathrm{A})$, fertirrigação parcial (B), fertirrigação parcial e solo coberto (C), fertirrigação total (D), e fertirrigação total e solo coberto $(E)^{1}$.

\begin{tabular}{ccccc}
\hline Tratamento & $\begin{array}{c}\mathrm{U} \\
\left(\text { dag. } \mathrm{kg}^{-1}\right)\end{array}$ & \multicolumn{1}{c}{ Tmáx } & \multicolumn{1}{c}{ Tmín } & \multicolumn{1}{c}{$\Delta \mathrm{T}$} \\
\hline A & $21,19 \mathrm{~b}$ & $26,5 \mathrm{~b}$ & $23,2 \mathrm{~b}$ & $3,3 \mathrm{a}$ \\
$\mathrm{B}$ & $21,83 \mathrm{~b}$ & $26,3 \mathrm{~b}$ & $23,3 \mathrm{~b}$ & $3,0 \mathrm{ab}$ \\
$\mathrm{C}$ & $23,36 \mathrm{a}$ & $26,7 \mathrm{ab}$ & $24,4 \mathrm{a}$ & $2,3 \mathrm{c}$ \\
$\mathrm{D}$ & $21,82 \mathrm{~b}$ & $26,4 \mathrm{~b}$ & $23,2 \mathrm{~b}$ & $3,2 \mathrm{a}$ \\
$\mathrm{E}$ & $23,70 \mathrm{a}$ & $27,0 \mathrm{a}$ & $24,3 \mathrm{a}$ & $2,7 \mathrm{bc}$ \\
\hline
\end{tabular}

${ }^{1}$ Médias seguidas da mesma letra na vertical não diferem estatisticamente entre si a 5\% de probabilidade pelo teste de Duncan. cobertura com plástico manteve a umidade e as temperaturas máxima e mínima do solo mais elevadas (Tabela 2). Entretanto, essas variações não apresentaram reflexos significativos na produção de tomate. As temperaturas mínima e máxima dos solos com cobertura de plástico superaram em 0,5 e $1,0^{\circ} \mathrm{C}$,
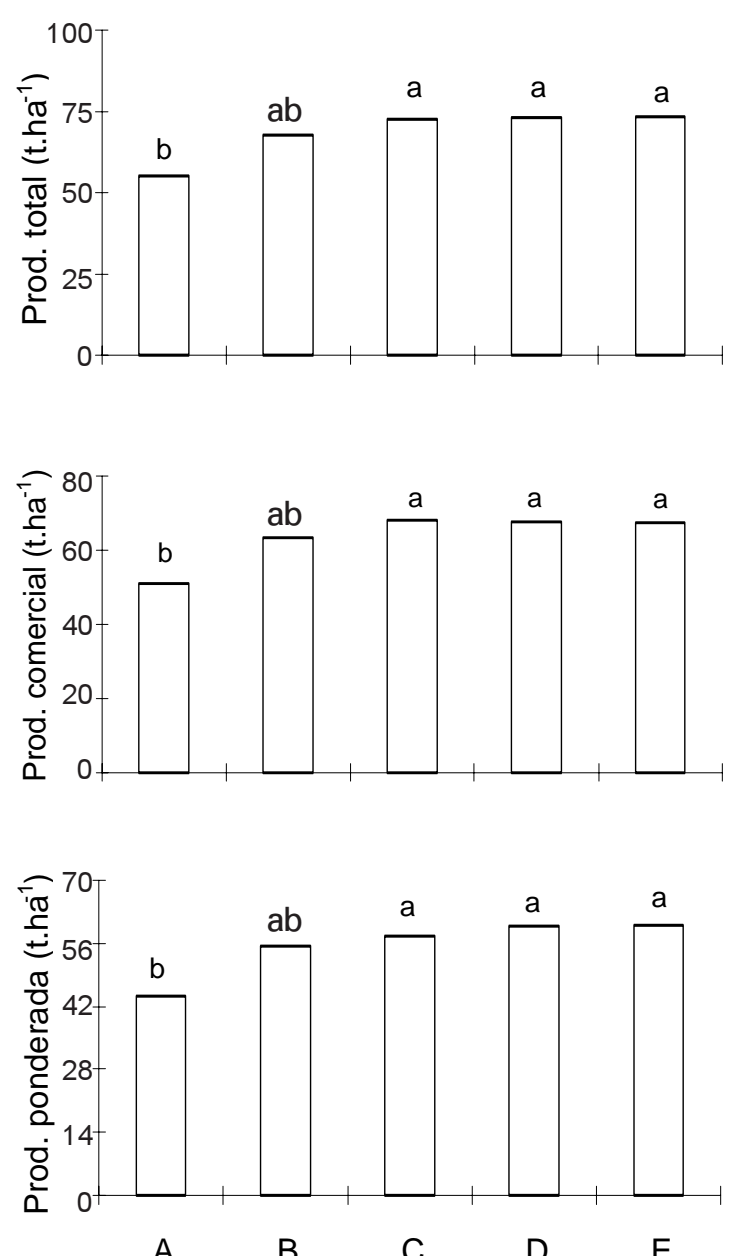

Tratamentos

FIG. 1. Produções total, comercial e ponderada de tomate nos tratamentos: testemunha (A), fertirrigação parcial (B), fertirrigação parcial e solo coberto (C), fertirrigação total (D), e fertirrigação total e solo coberto (E). Barras com letras iguais não diferem estatisticamente entre si a $5 \%$ de probabilidade pelo teste de Duncan. 
respectivamente, às dos solos sem cobertura; tais incrementos, no entanto, poderiam ser importantes em época de menores temperaturas, uma vez que, nessas condições, o desenvolvimento da planta e a absorção de nutrientes poderiam ser aumentados, conforme verificado por Martin \& Wilcox (1963).

Em geral, as produções de frutos de cada classe comercial, extra AA, extra A e extra, foram mais elevadas com a fertirrigação e com o uso do sistema de gotejamento do que com a aplicação manual do $\mathrm{K}$ e irrigação por aspersão no solo com mangueira (Tabela 3).

A produção não-comercial e os índices de precocidade das produções comerciais e totais não foram influenciados pelos tratamentos (Tabela 3), apesar de Wien \& Minotti (1988) afirmarem que colheitas mais precoces de tomate possam ser obtidas com o uso da cobertura plástica do solo. Em todos os tratamentos, a incidência de podridão apical do fruto foi praticamente inexistente.

Por ocasião do florescimento do sexto cacho de

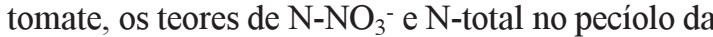
folha adjacente a ele não foram influenciados pelos tratamentos (Tabela 4). No pecíolo da folha completamente expandida, coletado na mesma data, ne-

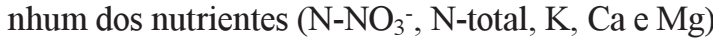
e a relação $\mathrm{K} /(\mathrm{Ca}+\mathrm{Mg})$ foi influenciado pelos tratamentos. Os teores de $\mathrm{N}^{-\mathrm{NO}_{3}}{ }^{-}$, N-total, $\mathrm{K}, \mathrm{Ca}$ e $\mathrm{Mg}$ no pecíolo da folha adjacente ao sexto cacho, e no pecíolo da folha completamente expandida, encon- tram-se dentro da faixa considerada por Reuter \& Robinson (1986) e Jones Junior et al. (1991) como suficientes para a cultura do tomateiro. Também, os teores de K no pecíolo da folha adjacente e no pecíolo da folha completamente expandida foram, em todos os tratamentos, maiores que o nível crítico citado por Jones Junior et al. (1991), o que indica o suprimento adequado deste elemento para as plantas nos diversos tratamentos.

As condutividades elétricas das soluções do solo, nas profundidades de $0-10$ e $10-20 \mathrm{~cm}$, foram maiores nas parcelas com cobertura de plástico do que nas parcelas sem cobertura (Tabela 5). Nas profundidades de 20-30, 30-40 e 40-50 cm, os efeitos dos tratamentos sobre a condutividade elétrica foram menos evidentes; porém, nas parcelas com cobertura de plástico, as condutividades elétricas foram quase sempre maiores do que nas sem cobertura. Este fato pode estar relacionado a maior mineralização da matéria orgânica, a menor lixiviação de nutrientes e a menor perda de $\mathrm{N}$ por volatilização. Com exceção dos tratamentos em que o solo foi coberto com plástico, nos quais as condutividades elétricas aparentemente decresceram em profundidade, não houve acúmulo de sais até $50 \mathrm{~cm}$ de profundidade, o que indica que o aumento da condutividade elétrica proporcionado pela cobertura de plástico do solo poderia estar mais relacionado à mineralização da matéria orgânica e à menor perda por volatilização de $\mathrm{N}$ do que à lixiviação de nutrientes nas parcelas sem cobertura.

TABELA 3. Produção classificada de frutos de tomate e índices de precocidade nos tratamentos: testemunha (A), fertirrigação parcial (B), fertirrigação parcial e solo coberto (C), fertirrigação total (D), e fertirrigação total e solo coberto $(E)^{1}$.

\begin{tabular}{clccccc}
\hline $\begin{array}{c}\text { Trata- } \\
\text { mento }\end{array}$ & Extra AA & Extra A & Extra & PNC $^{2}$ & IPC $^{3}$ & IPT $^{3}$ \\
\hline A & $37,74 \mathrm{~b}$ & $8,77 \mathrm{~b}$ & $4,68 \mathrm{~b}$ & $3,77 \mathrm{a}$ & $0,72 \mathrm{a}$ & $0,74 \mathrm{a}$ \\
$\mathrm{B}$ & $47,74 \mathrm{ab}$ & $10,70 \mathrm{ab}$ & $4,89 \mathrm{ab}$ & $4,21 \mathrm{a}$ & $0,89 \mathrm{a}$ & $0,91 \mathrm{a}$ \\
$\mathrm{C}$ & $51,62 \mathrm{a}$ & $11,60 \mathrm{a}$ & $4,93 \mathrm{ab}$ & $4,56 \mathrm{a}$ & $0,76 \mathrm{a}$ & $0,80 \mathrm{a}$ \\
$\mathrm{D}$ & $53,14 \mathrm{a}$ & $9,79 \mathrm{ab}$ & $4,68 \mathrm{~b}$ & $5,19 \mathrm{a}$ & $0,78 \mathrm{a}$ & $0,83 \mathrm{a}$ \\
$\mathrm{E}$ & $48,73 \mathrm{a}$ & $12,28 \mathrm{a}$ & $6,33 \mathrm{a}$ & $5,76 \mathrm{a}$ & $0,88 \mathrm{a}$ & $0,92 \mathrm{a}$ \\
\hline
\end{tabular}

${ }^{1}$ Médias seguidas da mesma letra na vertical não diferem estatisticamente entre si a 5\% de probabilidade pelo teste de Duncan.

${ }^{2}$ Produção não comercial.

3 Índice de precocidade da produção comercial (IPC) e da total (IPT) 
Os valores de $\mathrm{pH}$ do solo, nas profundidades de 0-10 e 10-20 cm, foram menores nas parcelas com cobertura de plástico do que nas parcelas sem cobertura (Tabela 5); o menor $\mathrm{pH}$ no solo com cobertura de plástico poderia estar relacionado à maior nitrificação do amônio advindo da matéria orgânica do solo e da uréia aplicada na adubação, em razão da maior conservação de umidade nos períodos mais secos e menor encharcamento nos períodos de chuvas intensas (Parchomchuk et al., 1993). Nas profundidades de 30-40 e 40-50 cm o pH não foi influenciado pelos tratamentos.

Em geral, os teores de $\mathrm{N}-\mathrm{NH}_{4}{ }^{+}$no solo não foram influenciados pelos tratamentos (Tabela 5) e, aparentemente, foram mais baixos na camada de 0-10 cm, onde a atividade biológica é mais intensa, aumentando moderamente nas camadas mais profundas. $\mathrm{O} \mathrm{N}-\mathrm{NH}_{4}{ }^{+}$pode causar a acidificação do solo, aumentar a solubilidade do $\mathrm{Ca}$ e do $\mathrm{Mg}$, e deslocar estes nutrientes e o $\mathrm{K}$ do complexo de troca. No caso da irrigação localizada, a aplicação do $\mathrm{N}-\mathrm{NH}_{4}{ }^{+}$pode também facilitar a movimentação de $\mathrm{Ca}, \mathrm{Mg}$ e K para as extremidades do bulbo molhado (Parchomchuk et al., 1993).

Os teores de $\mathrm{N}$ inorgânico total (obtido pela soma de $\mathrm{N}-\mathrm{NH}_{4}{ }^{+}+\mathrm{N}^{-} \mathrm{NO}_{3}{ }^{-}$), nas profundidades de 0-10 e
$10-20 \mathrm{~cm}$, foram maiores nas parcelas com cobertura de plástico do que nas parcelas sem cobertura (Tabela 5). Este fato pode estar associado a maior mineralização da matéria orgânica e menores perdas de N por desnitrificação nas parcelas com cobertura de plástico do solo, em face do encharcamento a que o solo foi submetido nas parcelas não cobertas com plástico, em razão das fortes chuvas, conforme verificado por Muirhead et al. (1985). Nas profundidades de $20-30,30-40$ e $40-50 \mathrm{~cm}$, as variações nos teores de $\mathrm{N}$ do solo não mostraram tendências definidas em função dos tratamentos. Com exceção dos solos com cobertura plástica, onde houve acúmulo nas primeiras camadas superficiais, 0 teor de $\mathrm{N}$ total aparentemente aumentou em profundidade, concentrando-se principalmente na profundidade de 40-50 cm

Os teores de $\mathrm{Ca}$ e $\mathrm{Mg}$, principalmente na profundidade de $0-10 \mathrm{~cm}$, foram mais elevados nos tratamentos com cobertura de plástico do que nos sem cobertura (Tabela 5). Este fato pode estar relacionado a maior mineralização da matéria orgânica ou a maior solubilização do calcário dolomítico aplicado imediatamente antes do transplante, em razão da maior redução do $\mathrm{pH}$. Nas demais profundidades, os teores de $\mathrm{Ca}$ e $\mathrm{Mg}$ não foram influenciados pelos trata-

TABELA 4. Teores de nutrientes e relações iônicas nos pecíolos das folhas adjacentes ao 6o cacho e completamente expandida nos tratamentos: testemunha (A), fertirrigação parcial (B), fertirrigação parcial e solo coberto (C), fertirrigação total (D), e fertirrigação total e solo coberto (E) ${ }^{\mathbf{1}}$.

\begin{tabular}{|c|c|c|c|c|c|c|c|}
\hline $\begin{array}{l}\text { Trata- } \\
\text { mento }\end{array}$ & N-orgânico & $\mathrm{N}-\mathrm{NO}_{3}^{-}$ & $\begin{array}{l}\text { N-total } \\
2 \\
\text { (dag.l }\end{array}$ & $\mathrm{K}$ & $\mathrm{Ca}$ & $\mathrm{Mg}$ & $\mathrm{K} /(\mathrm{Ca}+\mathrm{Mg})$ \\
\hline \multicolumn{8}{|c|}{ Pecíolo da folha adjacente ao $6^{\circ}$ cacho } \\
\hline $\mathrm{A}$ & $1,36 \mathrm{a}$ & $0,20 \mathrm{a}$ & $1,56 \mathrm{a}$ & $5,59 \mathrm{ab}$ & $1,81 b$ & $0,42 \mathrm{ab}$ & $2,53 \mathrm{a}$ \\
\hline B & $1,15 b$ & $0,21 \mathrm{a}$ & $1,37 \mathrm{a}$ & $5,69 \mathrm{a}$ & $1,78 b$ & $0,36 \mathrm{~b}$ & $2,69 \mathrm{a}$ \\
\hline $\mathrm{C}$ & $1,19 \mathrm{ab}$ & $0,21 \mathrm{a}$ & $1,40 \mathrm{a}$ & $5,50 \mathrm{ab}$ & $1,83 b$ & $0,41 \mathrm{ab}$ & $2,49 \mathrm{a}$ \\
\hline $\mathrm{D}$ & $1,26 \mathrm{ab}$ & $0,19 \mathrm{a}$ & $1,45 \mathrm{a}$ & $5,50 \mathrm{ab}$ & $1,88 b$ & $0,43 \mathrm{ab}$ & $2,52 \mathrm{a}$ \\
\hline $\mathrm{E}$ & $1,22 \mathrm{ab}$ & $0,24 \mathrm{a}$ & $1,46 \mathrm{a}$ & $4,93 b$ & $2,32 \mathrm{a}$ & $0,55 \mathrm{a}$ & $1,78 b$ \\
\hline \multicolumn{8}{|c|}{ Pecíolo da folha completamente expandida } \\
\hline $\mathrm{A}$ & $1,68 b$ & $0,35 \mathrm{a}$ & $2,03 \mathrm{a}$ & $5,08 \mathrm{a}$ & $3,29 a$ & $0,97 \mathrm{a}$ & $1,23 \mathrm{a}$ \\
\hline B & $1,74 a b$ & $0,28 \mathrm{a}$ & $2,03 \mathrm{a}$ & $4,78 \mathrm{a}$ & $3,22 \mathrm{a}$ & $0,91 \mathrm{a}$ & $1,16 \mathrm{a}$ \\
\hline $\mathrm{C}$ & $2,02 \mathrm{a}$ & $0,34 \mathrm{a}$ & $2,36 \mathrm{a}$ & $5,39 a$ & $3,49 a$ & $1,00 \mathrm{a}$ & $1,20 \mathrm{a}$ \\
\hline $\mathrm{D}$ & $1,72 \mathrm{ab}$ & $0,32 \mathrm{a}$ & $2,04 \mathrm{a}$ & $5,11 \mathrm{a}$ & $3,24 \mathrm{a}$ & $0,90 \mathrm{a}$ & $1,24 \mathrm{a}$ \\
\hline $\mathrm{E}$ & $1,75 \mathrm{ab}$ & $0,30 \mathrm{a}$ & $2,04 \mathrm{a}$ & $5,38 \mathrm{a}$ & $3,17 \mathrm{a}$ & $0,87 \mathrm{a}$ & $1,53 \mathrm{a}$ \\
\hline
\end{tabular}


TABELA 5. Condutividade elétrica (CE), pH e teores de nutrientes no solo nos tratamentos: testemunha (A), fertirrigação parcial (B), fertirrigação parcial e solo coberto (C), fertirrigação total (D), e fertirrigação total e solo coberto (E) em diferentes profundidades do solo (P) ${ }^{1}$.

\begin{tabular}{|c|c|c|c|c|c|c|c|}
\hline $\begin{array}{l}\text { Profundidade } \\
(\mathrm{cm})\end{array}$ & $\begin{array}{l}\text { Trata- } \\
\text { mento }\end{array}$ & $\begin{array}{c}\mathrm{CE} \\
\left(\mathrm{dS} . \mathrm{m}^{-1}\right) \\
\end{array}$ & $\mathrm{pH}$ & $\begin{array}{c}\mathrm{N}-\mathrm{NH}_{4}{ }^{+} \\
----(\mathrm{mg} . \mathrm{dm} \\
\end{array}$ & $\begin{array}{l}\text { N-total } \\
\left.\mathrm{T}^{-3}\right)^{2}---- \\
\end{array}$ & $\begin{array}{c}\mathrm{Ca}^{2+} \\
-----(\mathrm{cmo} \\
\end{array}$ & $\begin{array}{l}\mathrm{Mg}^{2+} \\
\left.\mathrm{dm}^{-3}\right)\end{array}$ \\
\hline \multirow[t]{5}{*}{$0-10$} & $\mathrm{~A}$ & $0,61 b$ & $5,55 \mathrm{a}$ & $9,49 \mathrm{a}$ & $12,94 b$ & $5,21 \mathrm{~b}$ & $0,98 \mathrm{bc}$ \\
\hline & B & $0,58 \mathrm{~b}$ & $5,59 \mathrm{a}$ & $9,61 \mathrm{a}$ & $16,19 b$ & $5,39 \mathrm{~b}$ & $0,94 b c$ \\
\hline & $\mathrm{C}$ & $2,10 \mathrm{a}$ & $5,28 \mathrm{ab}$ & $18,81 \mathrm{a}$ & $51,88 \mathrm{a}$ & $6,91 \mathrm{a}$ & $1,19 \mathrm{a}$ \\
\hline & D & $0,59 \mathrm{~b}$ & $5,40 \mathrm{ab}$ & $8,71 \mathrm{a}$ & $13,91 \mathrm{~b}$ & $4,60 \mathrm{~b}$ & $0,88 \mathrm{c}$ \\
\hline & $\mathrm{E}$ & $2,01 \mathrm{a}$ & $5,23 b$ & $13,32 \mathrm{a}$ & $35,48 \mathrm{ab}$ & $7,30 \mathrm{a}$ & $1,06 \mathrm{ab}$ \\
\hline \multirow[t]{5}{*}{$10-20$} & A & $0,64 b$ & $5,30 \mathrm{a}$ & $27,60 \mathrm{a}$ & $40,95 b$ & $4,84 \mathrm{a}$ & $0,84 b c$ \\
\hline & B & $0,59 b$ & $5,27 \mathrm{a}$ & $32,07 \mathrm{a}$ & $45,44 b$ & $4,58 \mathrm{a}$ & $0,78 \mathrm{c}$ \\
\hline & $\mathrm{C}$ & $1,45 \mathrm{a}$ & $4,91 \mathrm{~b}$ & $26,27 \mathrm{a}$ & $71,66 \mathrm{a}$ & $4,77 \mathrm{a}$ & $0,98 \mathrm{a}$ \\
\hline & D & $0,60 \mathrm{~b}$ & $5,21 \mathrm{a}$ & $29,50 \mathrm{a}$ & $44,09 b$ & $4,44 a$ & $0,81 \mathrm{c}$ \\
\hline & $\mathrm{E}$ & $1,36 \mathrm{a}$ & $4,98 \mathrm{~b}$ & $35,56 a$ & $74,75 \mathrm{a}$ & $4,88 \mathrm{a}$ & $0,94 \mathrm{ab}$ \\
\hline \multirow[t]{5}{*}{$20-30$} & A & $0,65 b$ & $4,78 \mathrm{a}$ & $17,20 \mathrm{a}$ & $39,47 \mathrm{ab}$ & $3,52 \mathrm{a}$ & $0,69 a$ \\
\hline & B & $0,57 \mathrm{~b}$ & $4,75 \mathrm{ab}$ & $21,20 \mathrm{a}$ & $35,76 \mathrm{ab}$ & $3,30 \mathrm{a}$ & $0,62 \mathrm{a}$ \\
\hline & $\mathrm{C}$ & $0,95 \mathrm{a}$ & $4,59 \mathrm{~b}$ & $18,43 \mathrm{a}$ & $35,51 \mathrm{ab}$ & $3,23 \mathrm{a}$ & $0,64 a$ \\
\hline & $\mathrm{D}$ & $0,59 b$ & $4,75 \mathrm{ab}$ & $11,69 a$ & $21,15 b$ & $3,37 \mathrm{a}$ & $0,62 \mathrm{a}$ \\
\hline & E & $0,76 \mathrm{~b}$ & $4,60 \mathrm{~b}$ & $21,84 \mathrm{a}$ & $42,77 \mathrm{a}$ & $3,39 a$ & $0,68 \mathrm{a}$ \\
\hline \multirow[t]{5}{*}{$30-40$} & A & $0,65 \mathrm{ab}$ & $4,78 \mathrm{a}$ & $20,46 a$ & $41,29 a$ & $3,34 \mathrm{a}$ & $0,73 a$ \\
\hline & B & $0,54 b$ & $4,70 \mathrm{a}$ & $24,27 \mathrm{a}$ & $38,00 \mathrm{a}$ & $3,08 \mathrm{a}$ & $0,68 \mathrm{a}$ \\
\hline & C & $0,83 \mathrm{a}$ & $4,73 \mathrm{a}$ & $32,49 a$ & $58,03 \mathrm{a}$ & $3,08 \mathrm{a}$ & $0,65 \mathrm{a}$ \\
\hline & $\mathrm{D}$ & $0,55 b$ & $4,58 \mathrm{a}$ & $27,10 \mathrm{a}$ & $41,50 \mathrm{a}$ & $3,43 a$ & $0,75 \mathrm{a}$ \\
\hline & $\mathrm{E}$ & $0,65 \mathrm{ab}$ & $4,60 \mathrm{a}$ & $18,78 \mathrm{a}$ & $40,73 a$ & $3,34 \mathrm{a}$ & $0,65 \mathrm{a}$ \\
\hline \multirow[t]{5}{*}{$40-50$} & A & $0,59 \mathrm{ab}$ & $5,10 \mathrm{a}$ & $41,87 \mathrm{ab}$ & $61,44 a b c$ & $4,22 \mathrm{a}$ & $0,61 \mathrm{a}$ \\
\hline & $\mathrm{B}$ & $0,51 \mathrm{~b}$ & $4,95 \mathrm{a}$ & $41,96 a b$ & $56,47 \mathrm{bc}$ & $3,98 \mathrm{a}$ & $0,58 \mathrm{a}$ \\
\hline & C & $0,62 \mathrm{a}$ & $4,86 a$ & $55,18 \mathrm{a}$ & $82,31 \mathrm{a}$ & $3,83 \mathrm{a}$ & $0,53 a$ \\
\hline & $\mathrm{D}$ & $0,52 \mathrm{ab}$ & $4,96 \mathrm{a}$ & $34,23 b$ & $47,64 \mathrm{c}$ & $3,96 a$ & $0,65 a$ \\
\hline & E & $0,57 \mathrm{ab}$ & $5,00 \mathrm{a}$ & $46,21 \mathrm{ab}$ & $73,40 \mathrm{ab}$ & $4,49 a$ & $0,58 \mathrm{a}$ \\
\hline
\end{tabular}

${ }^{1}$ Em cada profundidade, médias seguidas da mesma letra na vertical não diferem estatisticamente entre si a 5\% de probabilidade pelo teste de Duncan. ${ }^{2} \mathrm{~N}-$ total $=\mathrm{N}^{-\mathrm{NO}_{3}}{ }^{-}+\mathrm{N}-\mathrm{NH}_{4}^{+}$.

mentos. Nas parcelas sem cobertura de plástico, aparentemente, não houve acúmulo de $\mathrm{Ca}$ e de $\mathrm{Mg}$ em profundidade, o que evidencia a não-lixiviação destes nutrientes.

Os teores de $\mathrm{N}_{-} \mathrm{NO}_{3}{ }^{-}$no solo, nas profundidades de $0-10$ e $10-20 \mathrm{~cm}$, foram maiores nas parcelas com cobertura de plástico do que nas sem cobertura (Fig. 2). Este incremento no teor de $\mathrm{N}^{-\mathrm{NO}_{3}}{ }^{-}$poderia ser atribuído principalmente ao aumento da taxa de mineralização da matéria orgânica do solo, decorrente do aumento da temperatura e umidade no solo com cobertura de plástico (Tabela 2) e, talvez, às menores perdas de $\mathrm{N}-\mathrm{NO}_{3}{ }^{-}$por denitrificação em face da ocorrência de encharcamento do solo sem a cobertura de plástico, nos períodos de chuvas intensas (Steduto, 1984; Muirhead et al., 1985). Na profundidade de $20-30 \mathrm{~cm}$, o teor de $\mathrm{N}^{-\mathrm{NO}_{3}}{ }^{-}$não foi influenciado pelos tratamentos, enquanto nas profundidades de $30-40$ e $40-50 \mathrm{~cm}$, os teores de $\mathrm{N}-\mathrm{NO}_{3}{ }^{-}$foram menores nos tratamentos $\mathrm{B}$ e $\mathrm{D}$, o que evidencia tendência de os teores de $\mathrm{N}^{-\mathrm{NO}_{3}}{ }^{-}$no solo coberto serem maiores do que no solo não coberto. Nos solos sem cobertura de plástico houve lixiviação de $\mathrm{N}^{-\mathrm{NO}_{3}}{ }^{-}$apenas na camada de $0-10 \mathrm{~cm}$ 


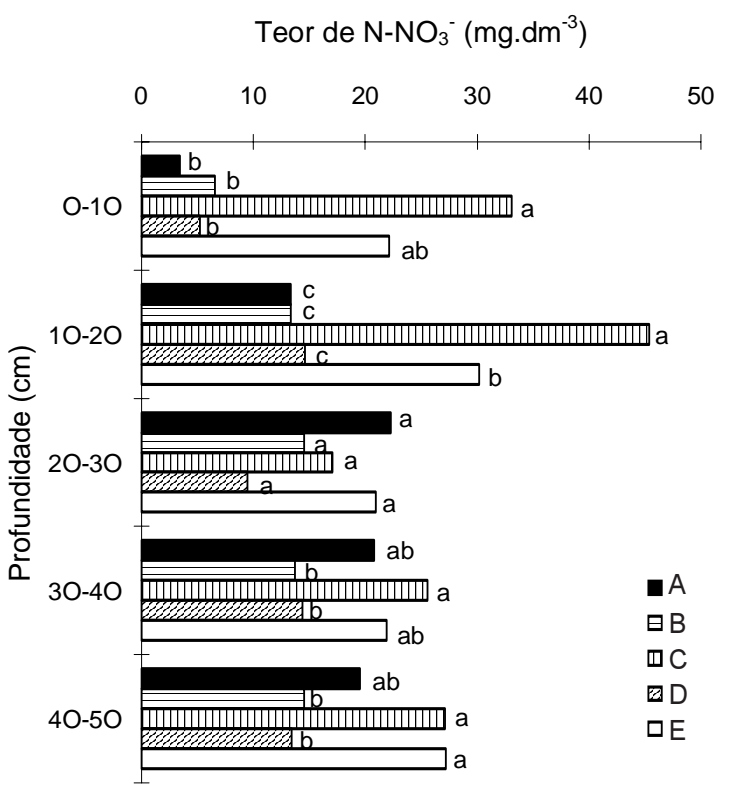

FIG. 2. Teor de N-NO ${ }_{3}^{-}$no solo nos tratamentos: testemunha (A), fertirrigação parcial (B), fertirrigação parcial e solo coberto (C), fertirrigação total (D), e fertirrigação total e solo coberto (E), em diferentes profundidades do solo. Em cada profundidade, barras com letras iguais não diferem estatisticamente entre si a $5 \%$ de probabilidade pelo teste de Duncan.

de profundidade; a não-lixiviação de $\mathrm{N}^{-\mathrm{NO}_{3}{ }^{+} \mathrm{em}}$ camadas mais profundas, conforme observado por Cook \& Sanders (1990), pode ser atribuída à presença de camadas adensadas ou compactadas, verificadas a partir de $20 \mathrm{~cm}$ de profundidade, limitando o fluxo de água e reduzindo a movimentação do $\mathrm{N}-\mathrm{NO}_{3}{ }^{-}$no solo no curto espaço de tempo da cultura.

Os teores de K, nas profundidades de $0-10,10-20$ e 30-40 cm, não foram influenciados pelos tratamentos (Fig. 3). O moderado decréscimo dos teores de $\mathrm{K}$ em profundidade evidencia a não-lixiviação deste nutriente das camadas superficiais, podendo-se atribuir tal fato à predominância de cargas

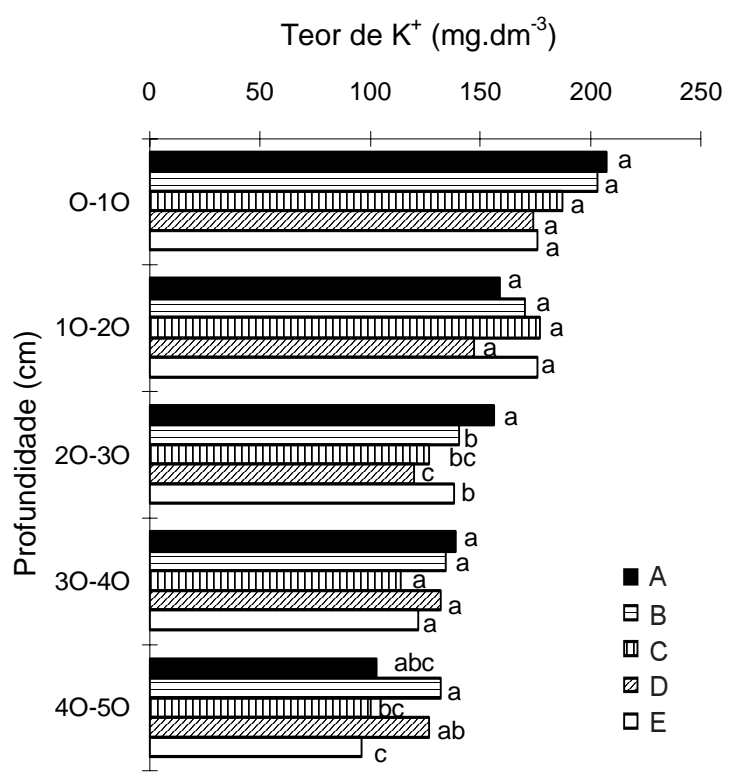

FIG. 3. Teor de $\mathrm{K}^{+}$no solo nos tratamentos: testemunha (A), fertirrigação parcial (B), fertirrigação parcial e solo coberto (C), fertirrigação total (D), e fertirrigação total e solo coberto (E), em diferentes profundidades do solo. Em cada profundidade, barras com letras iguais não diferem estatisticamente entre si a $5 \%$ de probabilidade pelo teste de Duncan.

eletronegativas no solo e à presença de camadas subjacentes adensadas.

\section{CONCLUSÕES}

1. Maiores produções de tomate são obtidas quando o potássio é aplicado por fertirrigação.

2. As produções de tomate não são influenciadas quando a fertirrigação é parcial ou total.

3. A cobertura do solo com plástico não influencia as produções de tomate.

4. Não há efeito dos métodos de aplicação do potássio e da cobertura plástica do solo sobre os teores de $\mathrm{N}_{-} \mathrm{NO}_{3}^{-}$, N-orgânico, $\mathrm{K}, \mathrm{Ca}$ e $\mathrm{Mg}$ nos pecíolos do tomateiro. 


\section{REFERÊNCIAS}

BAKI-ABDUL, A.; SPENCE, C. Black polyethylene mulch doubled yield of fresh-market field tomatoes. HortScience, Alexandria, v.27, n.7, p.787-789, 1992.

$\mathrm{BONOMO}, \mathrm{R}$. Análise da validade da equação utilizada para estimar a variação da concentração de fertilizante no tanque de derivação, em fertirrigação. Viçosa: UFV, 1995. 57p. Tese de Mestrado.

CATALDO, D.A.; HAROON, M; SCHRADER, L.E.; YOUNGS, V.L. Rapid colorimetric determination of nitrate in plant tissue by nitration of salicylic acid. Communications in Soil Science and Plant Analysis, New York, v.6, p.71-80, 1975

CLARK, G.A.; MAYNARD, D.N. Vegetable production on various bed widths using drip irrigation. Applied Engineering in Agriculture, St. Joseph, v.8, n.1, p.28-32, 1992

CLARK, G.A.; STANLEY, C.D; MAYNARD, D.N.; HOCMUTH, G.J.; HANLON, E.A.; HAMAN, D.Z. Water and fertilizer management of microirrigated fresh market tomatoes. Transactions of the ASAE, St. Joseph, v.34, n.2, p.429-435, 1991

COMISSÃO DE FERTILIDADE DO SOLO DO ESTADO DE MINAS GERAIS. Recomendações para uso de corretivos e fertilizantes em Minas Gerais: $4^{\mathrm{a}}$ aproximação. Lavras: CFSEMG, 1989. 76p.

COOK, W.P.; SANDERS, D.C. Fertilizer placement effects on soil nitrogen and use by drip-irrigated and plastic-mulched tomatoes. HortScience, Alexandria, v. 25, n.7, p.767-769, 1990

DANGLER, J.M; LOCASCIO, S.J. Yield of trickle-irrigated tomatoes as affected by time of $\mathrm{N}$ and $\mathrm{K}$ application. Journal of the American Society for Horticultural Science, St. Joseph, v.115, p.585-589, 1990 .

DEFELIPO, B.V.; RIBEIRO, A.C. Análise química do solo: metodologia. Viçosa: Universidade Federal de Viçosa, 1991. 17p. (Boletim de extensão, 29).

FISCHER, J.R. Water and nutrient requirements for drip-irrigated vegetables in humid regions. Gainesville: Institute of Food and Agricultural Sciences/Univ. of Florida, 1992. 17p. (Southern Cooperative Series Bulletin, 363).
GONÇALVES, A.C.A. Análise da perda de carga em tubos perfurados para irrigação, pela comparação entre o conceito de vazão fictícia e a metodologia proposta por Christiansen. Viçosa: UFV, 1988. 80p. Tese de Mestrado.

HARTZ, T.K. Water management in drip-irrigated vegetable production. In: AMERICAN SOCIETY HORTICULTURAL SCIENCE SEMINAR, 1994, Lexington. Proceedings... [S.1.]: American Society for Horticultural Science, 1994. p.12-15

JACKSON, M.L. Soil chemical analysis. Englewood Cliffs: Prentice Hall, 1958. 458p

JONES JUNIOR, J.B.; WOLF, B; MILLS, H.A. Plant analysis handbook: a practical sampling, preparation, analysis, and interpretation guide Athens: Micro-Macro Pub., 1991. 214p.

KHANIZADEH, S.; FANOUS, M.A. Mathematical indices for comparing small fruit crops for harvest time and trait similarity. HortScience, Alexandria, v.27, p.346-348, 1992.

LOCASCIO, S.J.; MYERS, J.M. Tomato response to plugmix, mulch and irrigation method. Proceedings of the Florida State Horticultural Science, Lake Alfred, v.85, p.126-130, 1974.

LOCASCIO, S.J.; MYERS, J.M.; FISKELL, J.G.A. Nitrogen application timing and source for drip irrigated tomatoes. In: INTERNATIONAL PLANT NUTRITION COLLOQUIUM, 9. 1982. Proceedings... [S.1.]: Warwick Univ., 1982. p.323-328.

LOCASCIO, S.J.; OLSON, S.M.; RHOADS, F.M. Water quantity and time of $\mathrm{N}$ and $\mathrm{K}$ application for trickleirrigated tomatoes. Journal of the American Society for Horticultural Science, St. Joseph, v.114, p.265-268, 1989

MALAVOLTA, E; VITTI, G.C.; OLIVEIRA, S.A. Avaliação do estado nutricional das plantas. Piracicaba: POTAFOS, 1989. 201p

MARTIN, G.C.; WILCOX, G.E. Critical soil temperature for tomato plant growth. Soil Science Society of America. Proceedings. Madison, v.27, p.565-567, 1963.

MUIRHEAD, W.A.; MELHUISH, F.M.; WHITE, R.J.G.; HIGGINS, M.L. Comparison of several nitrogen fertilizers applied in surface irrigation systems. II. Nitrogen transformations. Fertilizer Research, Dordrecht, v.8, p.49-68, 1985. 
PARCHOMCHUK, P.; NEILSEN, G.H.; HOGUE, E.J Effects of drip fertigation of $\mathrm{NH}_{4}-\mathrm{N}$ and $\mathrm{P}$ on soil $\mathrm{pH}$ and cation leaching. Canadian Journal of Soil Science, Ottawa, v.73, p.157-164, 1993.

PIMENTEL-GOMES, F. Curso de Estatística Experimental. 12.ed. Piracicaba: Nobel, 1987. 468p.

REUTER, D.J.; ROBINSON, J.B. (Eds.). Plant analysis: an interpretation manual. Melbourne: Inkata Press, 1986. $218 \mathrm{p}$.

SALVETTI, M.G. O polietileno na agropecuária brasileira. São Paulo: Poliolefinas, 1985. 154p.

SAMPAIO, R.A. Produção, qualidade dos frutos e teores de nutrientes no solo e no pecíolo do tomateiro, em função da fertirrigação potássica e da cobertura plástica do solo. Viçosa: UFV, 1996. 117p. Tese de Doutorado.

SMAJSTRLA, A.G.; LOCASCIO, S.J. Irrigation scheduling of drip-irrigated tomato using tensiometers and pan evaporation. Proceedings of the Florida State Horticultural Society, Lake Alfred, v.103, p.88-91, 1990.
SOARES, J.M. Sistemas de irrigação por mangueiras. Petrolina: Embrapa-CPTSA, 1986. 132p. (Embrapa-CPTSA. Circular técnica, 13).

STEDUTO, P. Fertigation. Rivista di agronomia, Bologna, v.18, n.1, p.3-20, 1984

TEDESCO, M.J.; VOLKWEISS, S.J.; BOHNEM, H. Análises de solo, plantas e outros materiais. Porto Alegre: UFRGS, 1985. n.p. (Boletim técnico, 5).

TSEKLEEV, G.; BOYADJIEVA, N.; SOLAKOV, Y. Influence of photo-selective mulch films on tomatoes in greenhouses. Plasticulture, Paris, v.95, p.45-49, 1993.

VOLPE, C.A.; CHURATA-MASCA, M.G.C. Manejo da irrigação em hortaliças: método do tanque classe "A". Jaboticabal: Funep, 1988. 20p.

WIEN, H.C.; MINOTTI, P.L. Increasing yield of tomatoes with plastic mulch and apex removal. Journal of the American Society for Horticultural Science, St. Joseph, v.113, n.3, p.342-347, 1988. 